\title{
Hypothermia inhibits the proliferation of bone marrow-derived mesenchymal stem cells and increases tolerance to hypoxia by enhancing SUMOylation
}

\author{
XIAOZHI LIU ${ }^{1-3}$, WENBO REN $^{3}$, ZHONGMIN JIANG $^{4}$, ZHIGUO SU $^{5}$, XIAOFANG MA $^{2}$, \\ YANXIA LI ${ }^{2}$, RONGCAI JIANG ${ }^{1}$, JIANNING ZHANG ${ }^{1}$ and XINYU YANG ${ }^{1}$ \\ ${ }^{1}$ Department of Neurosurgery, Tianjin Medical University General Hospital, Tianjin 300052; \\ ${ }^{2}$ Central Laboratory, and Departments of ${ }^{3}$ Neurology, ${ }^{4}$ Pathology and ${ }^{5}$ Neurosurgery, \\ The Fifth Central Hospital of Tianjin, Tianjin 300450, P.R. China
}

Received October 15, 2016; Accepted September 5, 2017

DOI: $10.3892 / \mathrm{ijmm} .2017 .3167$

\begin{abstract}
Hypothermia therapy has a positive effect on patients with severe brain injury. Recent studies have shown that mild hypothermia increases the survival of bone marrow-derived mesenchymal stem cells (BMSCs) in a hypoxic environment; however, the underlying mechanisms are not yet fully understood. Small ubiquitin-like modifiers (SUMOs) are sensitive to temperature stress reactions and are considered to exert a protective effect. In this study, we examined the protective effects of hypothermia on BMSCs in terms of SUMO protein modification. First, we found that mild hypothermia inhibited the proliferation and differentiation of BMSCs and increased cell tolerance to a hypoxic environment. Second, hypothermia significantly increased the levels of SUMO modification of multiple proteins in BMSCs. The knockdown of SUMO1/2/3 induced the rapid aging of the BMSCs, while the inhibition of the SUMO-conjugating enzyme, Ubc9, reduced cell proliferation and increased the proportion of BMSCs differentiating into nerve cells. Moreover, the tolerance of BMSCs to the hypoxic environment was significantly decreased. Lastly, we investigated 4 reported SUMO target proteins, anti-proliferating cell nuclear antigen, octamer-binding transcription factor 4 , p53 and hypoxia-inducible factor- $1 \alpha$, to confirm that SUMO modification was indeed involved in maintaining the proliferation, inhibiting differentiation and enhancing the resistance of BMSCs against adverse conditions. Taken together, our results indicate that the SUMO pathway is involved in the response to hypothermic stress, and that SUMOylation may be an important protective mechanism against hypothermia for the survival of BMSCs under unfavorable conditions.
\end{abstract}

Correspondence to: Professor Xinyu Yang, Department of Neurosurgery, Tianjin Medical University General Hospital, 154 Anshan Road, Heping, Tianjin 300052, P.R. China

E-mail: chnxyyang@163.com

Key words: small ubiquitin-like modifiers, SUMOylation, therapeutic hypothermia, stress, bone marrow-derived mesenchymal stem cells

\section{Introduction}

Brain injuries range in scope from mild to severe. Severe brain injuries result in permanent neurobiological damage that can produce lifelong deficits to varying degrees $(1,2)$. There are many different types of treatments available for patients with severe brain injury. Surgical treatment may be used to prevent secondary injury by helping to maintain blood flow and oxygen to the brain, and minimizing swelling and pressure (3-5). Intracranial pressure can be decreased by lowering or maintaining body temperature, elevating the head of the bed, keeping the person still and comfortable, ensuring proper breathing and applying hypertensive therapies $(1,2,4,6)$.

Therapeutic or protective hypothermia is an active treatment aimed at achieving and maintaining a specific body temperature for a specific duration of time to improve health outcomes during recovery following a period of suppressed blood flow to the brain $(7,8)$. Targeted temperature management is thought to prevent brain injury through several mechanisms, including decreasing the oxygen demand of the brain, reducing the production of neurotransmitters, such as glutamate and reducing free radicals that may damage the brain (7-10). Recent studies have indicated that hypothermia increases the survival of transplanted cells in a hypoxic environment $(11,12)$; however, the detailed mechanisms are poorly understood.

Small ubiquitin-like modifier (SUMO) proteins are a family of small proteins that are covalently attached to and detached from other proteins in cells to modify their function (13). SUMOylation is a post-translational modification involved in various cellular processes, such as nuclear-cytosolic transport, transcriptional regulation, apoptosis, maintaining protein stability, responding to stress and cell cycle progression (14-19). Recent studies have demonstrated that SUMO1/2/3 conjugation is markedly activated in the brain during deep to moderate hypothermia, and that hypothermia induces the nuclear translocation of the SUMO-conjugating enzyme, Ubc9 (20-22). These functions may lead to the development of novel strategies for preventive and therapeutic interventions with an aim of making neurons more resistant to an adverse environment. 
Bone marrow-derived mesenchymal stem cells (BMSCs) are multipotent stem cells capable of differentiating into numerous cell types, including fibroblasts, as well as cartilage, bone, muscle and brain cells (23-26). They secrete a large number of growth factors and cytokines that are critical for the repair of injured tissues $(23,24,27)$. In this study, we determined whether SUMOs and the SUMO cycle are involved in the effects of hypothermia on BMSCs and their role in an adverse environment.

This study found that BMSCs subjected to $24 \mathrm{~h}$ of low temperature conditions exhibited a low proliferative activity, almost no differentiation and a higher tolerance to a hypoxic environment. Similar to neurons exposed to low temperatures, the level of SUMOylation was markedly increased upon exposure of the BMSCs to hypothermic conditions. The knockdown of SUMO1/2/3 or the inhibition of the SUMO-conjugating enzyme, Ubc9, markedly increased the proportion of BMSCs differentiating into nerve cells in a hypoxic environment. Moreover, the tolerance of the cells to the adverse environment was significantly decreased. This study implies that SUMOylation induced by hypothermia is essential to maintaining the stemness and inhibiting the differentiation of BMSCs, and enhances resistance against adverse conditions. This study may provide the basis for a novel treatment option based on the SUMO pathway, such as drugs that induce endogenous SUMO expression or increase the supply of exogenous SUMO products.

\section{Materials and methods}

Animals and cell culture. To obtain BMSCs, 2 newborn male Sprague-Dawley rats were purchased from the Animal Center of the Cancer Institute of the Chinese Academy of Medical Sciences (Beijing, China). All experimental procedures were carried out according to the regulations and internal biosafety and bioethics guidelines of Tianjin Medical University and the Tianjin Municipal Science and Technology Commission. BMSCs were collected from the long bones of hind legs as previously described (28). Briefly, the long bones were dissected out, and bone marrow plugs were extracted from the bones by flushing the bone marrow cavity with complete culture medium. The cells were plated at $5 \times 10^{7}$ cells per $75-\mathrm{cm}^{2}$ culture flask and incubated at $37^{\circ} \mathrm{C}$ in a humidified atmosphere with $5 \% \mathrm{CO}_{2}$. Non-adherent cells were removed after $24 \mathrm{~h}$, and the culture medium was replaced every 3 days. Adherent cells reached $90-95 \%$ confluence within 10-15 days and were passaged with $0.25 \%$ trypsin (Invitrogen Life Technologies, Carlsbad, CA, USA) at a ratio of 1:3. Cells at passage 4 were characterized by flow cytometric analysis to detect the expression of the surface antigens, CD34, CD45, CD71 and CD105 (Abcam Trading Co. Ltd., Shanghai, China).

Exposure to hypothermia and hypoxia. To simulate hypothermic stress, the culture dishes loaded with BMSCs were placed in incubators for $24 \mathrm{~h}$ at $37^{\circ} \mathrm{C}$ (normal), $33^{\circ} \mathrm{C}$ (mild hypothermia), or $18^{\circ} \mathrm{C}$ (deep hypothermia) with $5 \% \mathrm{CO}_{2}$. To evaluate the effects of hypothermia on the tolerance of BMSCs to the adverse environment, culture medium without glucose, L-aspartic acid, L-glutamic acid or sodium pyruvate was equilibrated overnight in an anoxic chamber with $85 \% \mathrm{~N}_{2}, 10 \% \mathrm{H}_{2}$ and $5 \% \mathrm{CO}_{2}$. The cultures were transferred to the anoxic chamber and washed
3 times with anoxic medium. After 24 h of oxygen-glucose deprivation (OGD), the cells were transferred back to the incubator at $37^{\circ} \mathrm{C}$ with $5 \% \mathrm{CO}_{2}$ for an additional $24 \mathrm{~h}$.

Cell viability assay. Cell viability was determined by a 3-(4,5-dimethylthiazol-2-yl)-2,5-diphenyltetrazolium bromide (MTT) assay (Sigma-Aldrich, St. Louis, MO, USA) according to the manufacturer's instructions. Briefly, the BMSCs were seeded in 96-well plates at a density of $1 \times 10^{4}$ cells/ well and incubated as described above. Subsequently, $15 \mu \mathrm{l}$ of MTT solution ( $5 \mathrm{mg} / \mathrm{ml}$ in PBS) were added to each well. After $3 \mathrm{~h}$ of incubation at $37^{\circ} \mathrm{C}$, the culture medium was aspirated and $100 \mu \mathrm{l}$ of dimethyl sulfoxide were added. The absorbance was monitored using a spectrophotometer with a microplate reader at a wavelength of $595 \mathrm{~nm}$ (Bio-Rad Laboratories, Inc., Hercules, CA, USA).

Cell cycle analysis. The cells were collected by trypsinization, washed in phosphate-buffered saline (PBS), and fixed in $70 \%$ ethanol for $30 \mathrm{~min}$ at $4^{\circ} \mathrm{C}$. The cells were then treated with the DNA-binding dye propidium iodide $(50 \mu \mathrm{g} / \mathrm{ml})$ and RNase $(1 \mathrm{mg} / \mathrm{ml})$ for $30 \mathrm{~min}$ at $37^{\circ} \mathrm{C}$ in the dark. Finally, the cells were washed, and red fluorescence was analyzed by a FACSCalibur flow cytometer (BD Biosciences, San Jose, CA, USA) using a peak fluorescence gate to discriminate aggregates.

Detection of lactate dehydrogenase ( $L D H)$ activity. After harvesting the cells, the LD H content in the conditioned medium was measured by an enzyme-linked immunosorbent assay (ELI SA) using an LD H Activity Assay kit (cat. no. K726-500; BioVision, Inc., Milpitas, CA, USA) in accordance with the manufacturer's instructions.

Apoptosis assay. The BMSCs were seeded in 12-well plates and incubated as described above. The cells were mounted in PBS, immersed in permeabilization solution for $5 \mathrm{~min}$, incubated with $25 \mu 1$ TUNEL-labeling reaction mixture in a humidified box at $37^{\circ} \mathrm{C}$ for $60 \mathrm{~min}$ and then washed with PBS. Nuclei were counterstained with Hoechst 33258 at a dilution of 1:1,000. Cell apoptosis rate (number of apoptotic cells/total number of cells $\mathrm{x} 100 \%$ ) was quantified.

Detection of cell differentiation. To induce BMSCs to differentiate into neural cells, the BMSCs were cultured in $3 \%$ oxygen. Cell differentiation was then detected by the immunocytochemical analysis of markers for undifferentiated BMSCs (nestin) and differentiated BMSCs [glial fibrillary acidic protein (GFAP) for astrocytes and $\beta$-tubulin III for neurons]. Immunocytochemistry was performed as previously described (29). Cells in 96-well plates were fixed with $2 \%$ paraformaldehyde for $15 \mathrm{~min}$ at room temperature, treated with $5 \%$ normal goat serum (Vector Laboratories, Inc., Burlingame, CA, USA), and then stained with the following antibodies: anti-nestin (mouse monoclonal IgG1, 1:200, cat. no. ab11306), anti- $\beta$-tubulin III (mouse monoclonal IgG1, 1:500, cat. no. ab78078) and anti-GFAP (rabbit polyclonal, 1:200, cat. no. ab16997) (all from Abcam, Cambridge, MA, USA). The primary antibodies were detected with goat anti-mouse IgG-CruzFluor ${ }^{\text {TM }} 488$ (1:400; cat. no. sc-362257) or goat anti-mouse IgG-CruzFluor ${ }^{\mathrm{TM}} 594$ (1:200; cat. no. sc-362277) or 


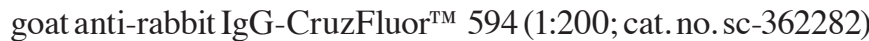
(all from Santa Cruz Biotechnology, Inc., Dallas, TX, USA) secondary antibodies at $37^{\circ} \mathrm{C}$ for $1 \mathrm{~h}$, followed by mounting and observation under a fluorescence microscope (Olympus DP70; Olympus Corp., Tokyo, Japan). The cells were counterstained with 4,6-diamidino-2-phenylindole (Vector Laboratories, Inc.). Images of 10 random fields were captured per well. Image-Pro Plus version 6.0 software (Media Cybernetics, Inc., Rockville, MD, USA) was used for image analysis.

Blockade of the SUMO pathway. Dominant negative mutant SUMO1/2/3 (siR-neg) plasmids or the same DNA fragment carrying siR-SUMO1/2/3 were subcloned into pCMV-Myc and then cloned into a lentiviral vector, pCDH-CMV-MCS-EF1-copGFP (System Biosciences, Mountain View, CA, USA). Transfection was performed using RNAifectin reagent (Applied Biological Materials) following the manufacturer's instructions. In brief, $1 \times 10^{5}$ BMSCs were seeded in 6-well plates and incubated overnight. Approximately 100 pmol siRNAs per well was used for transfection. In another group of BMSCs, $20 \mu \mathrm{M}$ spectomycin B1 (Nanjing Chemlin Chemical Co., Ltd., Nanjing, China) was added to the culture medium to block the effects of Ubc9 for $24 \mathrm{~h}$. The morphology of cells was observed under a high magnification microscope. When cell morphology showed increased cell volume, flattened, increased nucleus and nucleolar volume, it was regarded as cellular aging.

Western blot analysis. Total protein was extracted by a cell lysis solution. Western blot analysis was performed using 4-15\% SDS-PAGE gels (Bio-Rad Laboratories, Inc.). Following electrophoresis, the proteins were transferred onto polyvinylidene fluoride membranes (Bio-Rad Laboratories, Inc.). The membranes were blocked using Tris/HCl-buffered salt solution containing $0.1 \%$ Tween-20 and 5\% skim milk powder, and then incubated with antibodies to anti-SUMO1 (1:2,000; cat. no. ab133352), anti-SUMO2/3 (1:5,000; cat. no. ab3742) and anti-GFAP (1:2,000; cat. no. ab7260), anti-proliferating cell nuclear antigen (PCNA; 1:1,000; cat. no. ab18197), anti-octamerbinding transcription factor 4 (Oct4; 1:1,000; cat. no. ab18976) (all from Abcam), anti-p53 (1:2,000; cat. no. sc-47698) or antihypoxia-inducible factor- $1 \alpha$ (HIF-1 $\alpha ; 1: 2,500$; cat. no. sc-71247) (both from Santa Cruz Biotechnology, Inc.) overnight at $4^{\circ} \mathrm{C}$. The membranes were then washed 5 times in $0.1 \%$ TBST and incubated for $1 \mathrm{~h}$ with the secondary antibodies chicken antirabbit IgG conjugated to horseradish peroxidase (HRP) $(1: 2,000$; cat. no. sc-516087) or chicken anti-goat IgG-HRP $(1: 2,000$; cat. no. sc-516086) (both from Santa Cruz Biotechnology, Inc.). Labeled proteins were detected using a Super Signal protein detection kit (Pierce, Thermo Fisher Scientific, Inc., Waltham, MA, USA). The membranes were then stripped and reprobed with a goat anti- $\beta$-actin polyclonal primary antibody $(1: 1,000$; cat. no. sc-70319; Santa Cruz Biotechnology, Inc.). Changes in the levels of SUMO-conjugated proteins were evaluated using ImageJ image analysis software (NIH, Bethesda, MD, USA). The high molecular weight area in each lane was cropped and analyzed.

Detection of nuclear translocation. To observe whether hypothermia can induce the translocation of SUMO1 and
SUMO2/3 complexes from the cytoplasm to the nucleus in BMSCs, immunocytochemistry assay was performed. Briefly, the BMSCs were seeded in 6-well plates and incubated at $37^{\circ} \mathrm{C}$, $33^{\circ} \mathrm{C}$, or $18^{\circ} \mathrm{C}$ with $5 \% \mathrm{CO}_{2}$. After $24 \mathrm{~h}$, the cells were fixed with $2 \%$ paraformaldehyde for $15 \mathrm{~min}$ at room temperature, treated with 5\% normal goat serum (Vector Laboratories, Inc.), and then stained with anti-SUMO1 (1:200, cat. no. ab133352) and anti-SUMO2/3 (1:400, cat. no. ab81371) (both from Abcam). The SUMO1 antibodies were then detected with goat anti-rabbit IgG-CruzFluor ${ }^{\mathrm{TM}} 594$ (1:200; cat. no. sc-362282) and SUMO2/3 antibodies were detected with goat anti-mouse IgG-CruzFluor ${ }^{\mathrm{TM}} 488$ (1:400; cat. no. sc-362257) (all from Santa Cruz Biotechnology Inc.) at $37^{\circ} \mathrm{C}$ for $1 \mathrm{~h}$, followed by mounting and observation under a fluorescence microscope (Olympus DP70; Olympus Corp.).

Statistical analysis. All experiments were repeated at least 3 times. The results were analyzed using the Student's t-test or two-way analysis of variance (ANOVA). Data are expressed as the means \pm standard error of the mean. All tests were two-tailed, and the level of statistical significance was set at $\mathrm{p} \leq 0.05$. GraphPad Prism 6 software (GraphPad Software, Inc., La Jolla, CA, USA) was used for all statistical tests.

\section{Results}

Hypothermia inhibits the proliferation and differentiation of BMSCs, and increases their tolerance to hypoxia. To verify that the cells used in the following experiments were BMSCs, 4 proteins to identify BMSCs were detected by flow cytometry. The cells exhibited a high expression of the BMSC-specific proteins, CD71 and CD105, but did not express the hematopoietic stem cell-specific proteins, CD34 and CD45 (Fig. 1A). To observe the growth characteristics of BMSCs at a low temperature, we examined their proliferative activity, their ability to differentiate into neural cells and their tolerance to hypoxic environments. The results revealed the slow growth of BMSCs at a low temperature (Fig. 1B and C), and a decreased ability to differentiate into neural cells (Fig. 1D). These trends increased with the decreasing temperature. When the BMSCs were cultured under oxygen-deprived conditions for $24 \mathrm{~h}$, a large number of apoptotic cells was detected (Fig. 1E and F), which was accompanied by high levels of lactate dehydrogenase (LDH) release (Fig. 1G). Of note, the cell apoptotic rate and LDH release were significantly reduced at a low temperature (Fig. 1E-G). These results indicate that a low temperature environment reduces the proliferative activity of BMSCs, decreases their differentiation potential, and increases their tolerance to an adverse environment.

Hypothermia induces SUMOylation and SUMO nuclear transfer in BMSCs. Some studies have shown that SUMO1/2/3 conjugation is markedly activated in neurons at low temperatures $(20,21)$. Whether BMSCs have a similar response to low temperature stress as neurons is unknown. Our results revealed that exposure of the cells to a low temperature induced the modification of numerous target proteins by SUMOs, including SUMO1 and SUMO2/3 (Fig. 2A-D). The levels of SUMO1 and SUMO2/3 in the covalent binding state were increased significantly, while the levels of free SUMO1 and SUMO2/3 were decreased (Fig. 2A-D). One of the concomitant phenomena 
A

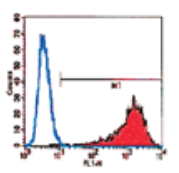

CD34

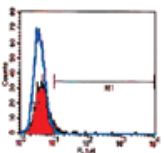

CD71

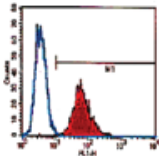

CD45

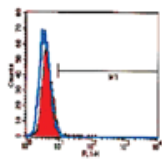

CD105
B

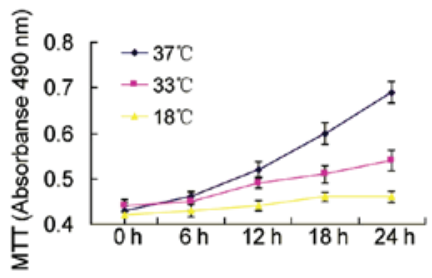

C
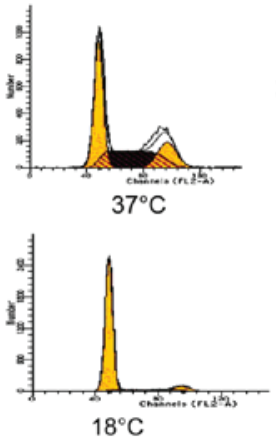

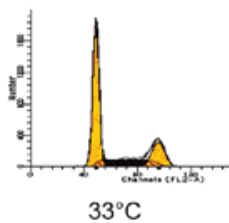

$33^{\circ} \mathrm{C}$

D

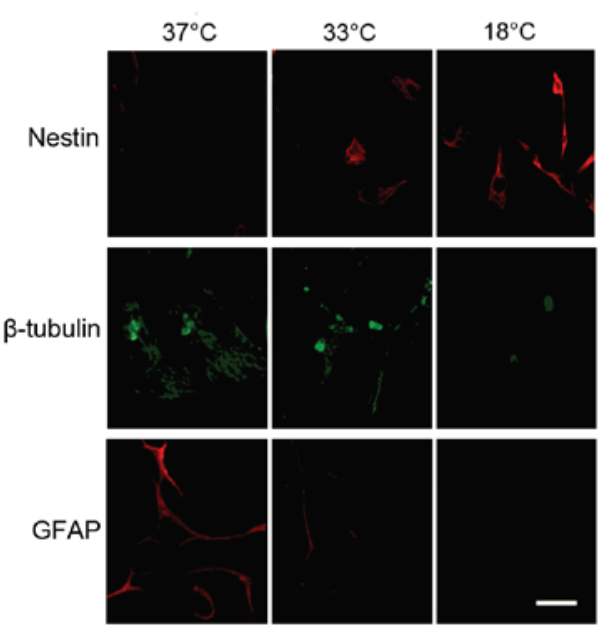

$\mathrm{E}$
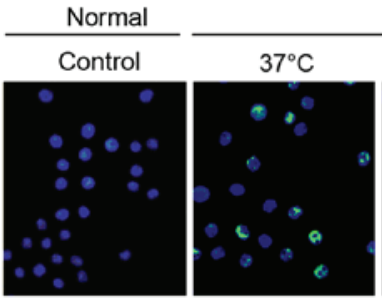

OGD

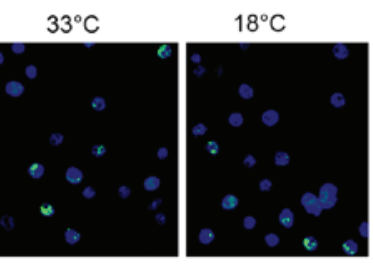

$\mathrm{F}$

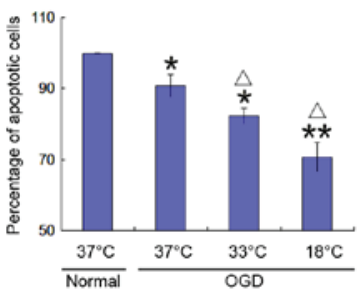

G

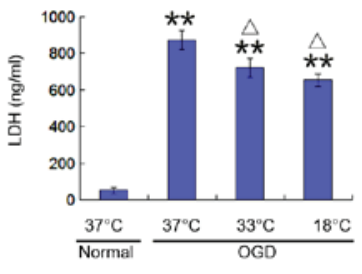

Figure 1. Hypothermia inhibits the proliferation and differentiation of bone marrow-derived mesenchymal stem cells (BMSCs) and increases their tolerance to hypoxia. (A) Detection of CD71, CD105 (BMSC markers), CD34 and CD45 (hematopoietic stem cell markers) in BMSCs by flow cytometry. (B) Growth curve of BMSCs. (C) Cell cycle analysis of BMSCs by flow cytometry. (D) Differentiation of BMSCs into nerve cells detected by immunofluorescence (scale bar, $25 \mu \mathrm{m}$ ). (E and F) Apoptosis detection by flow cytometry (scale bar, $50 \mu \mathrm{m}$ ). (G) Measurement of LDH content by enzyme-linked immunosorbent assay (ELISA). ${ }^{*} \mathrm{p}<0.05$ and ${ }^{* *} \mathrm{p}<0.01$, compared with the $37^{\circ} \mathrm{C}$ normal group; ${ }^{\circ} \mathrm{p}<0.05$, compared with the $37^{\circ} \mathrm{C}$ OGD group, .

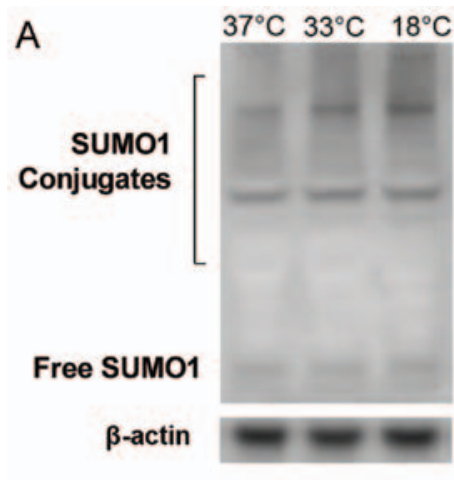

B

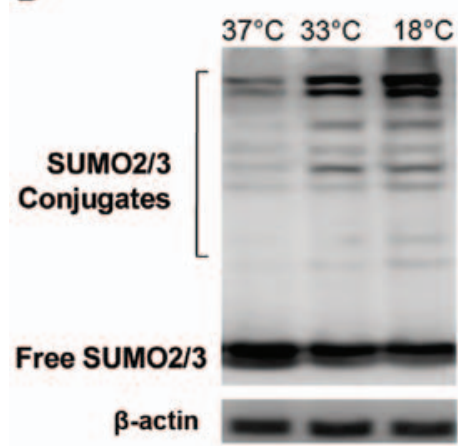

C

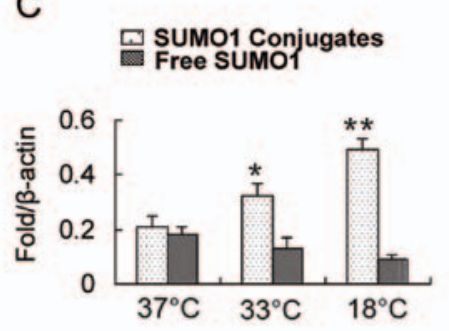

D

$\square$ SUMO2/3 Conjugates 口ree SUMO2/3

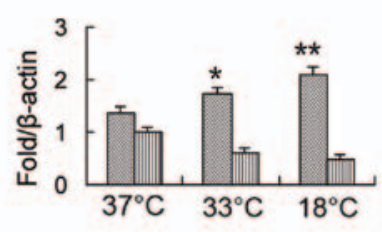

E
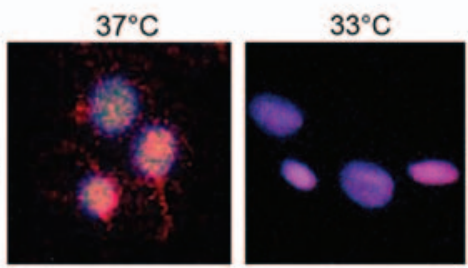

$18^{\circ} \mathrm{C}$

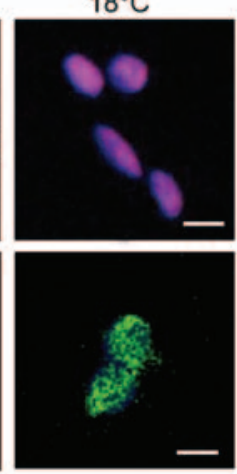

Figure 2. Hypothermia induces SUMOylation and small ubiquitin-like modifier (SUMO) nuclear transfer in bone marrow-derived mesenchymal stem cells (BMSCs). (A) Detection of SUMO1 levels in covalent and free states by western blot analysis. (B) Detection of SUMO2/3 levels in covalent and free states by western blot analysis. (C) Quantification of data in panel A. (D) Quantification of data in panel B. (E) A shift of SUMO1 and the SUMO2/3 complex from the cytoplasm to the nucleus was observed by immunofluorescence (scale bar, $10 \mu \mathrm{m}$ ). Red, SUMO1 protein; green, SUMO2/3 protein; blue, DAPI labeling of nuclei. * $\mathrm{p}<0.05$, $^{* *}$ p $<0.01$. 

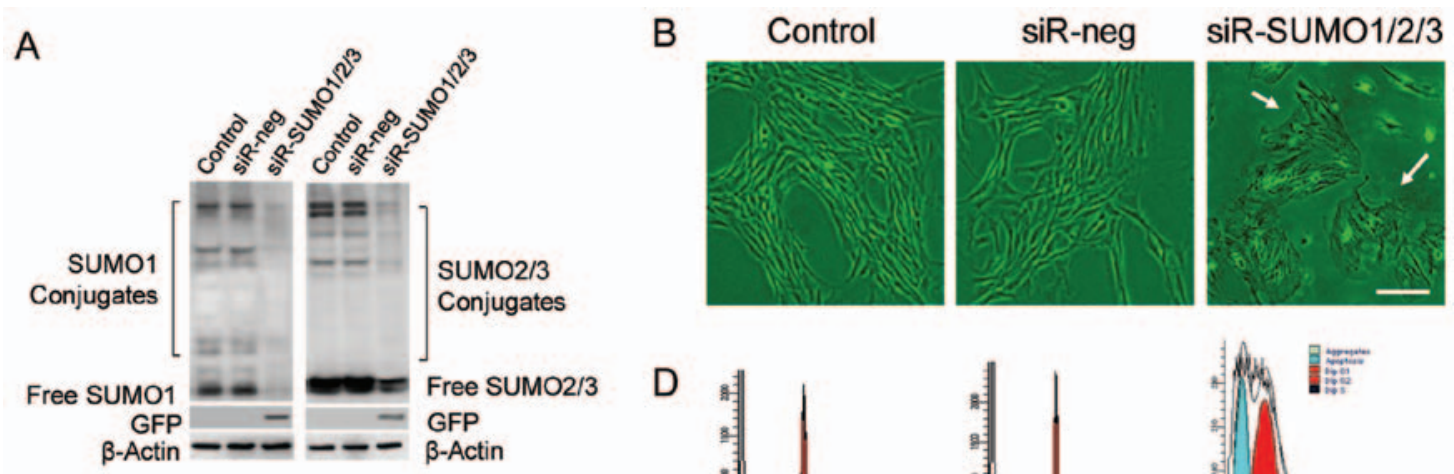
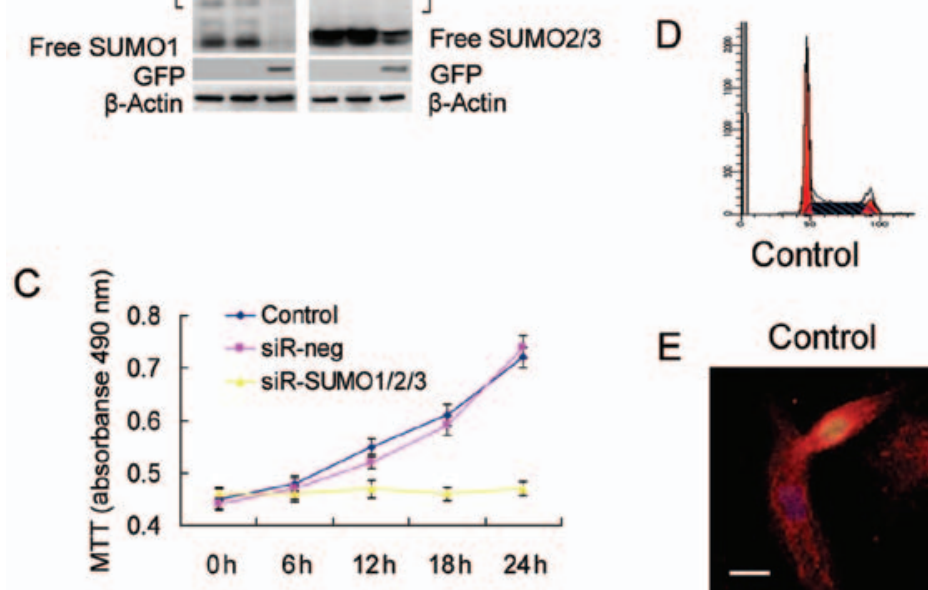

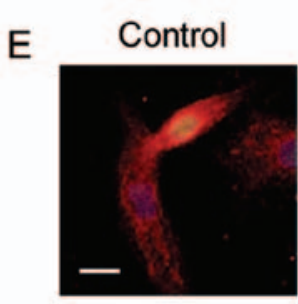

Nestin
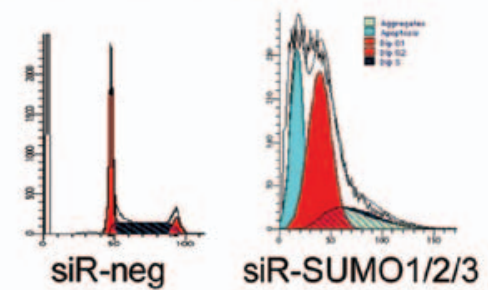

siR-SUMO1/2/3

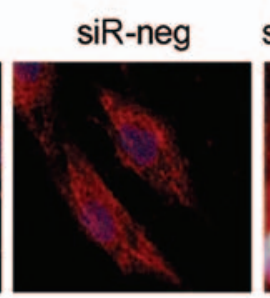

Nestin

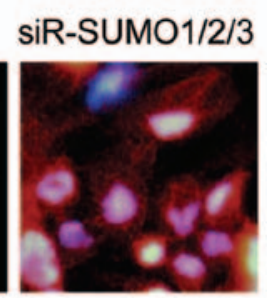

GFAP

Figure 3. Small ubiquitin-like modifiers (SUMOs) are essential for bone marrow-derived mesenchymal stem cell (BMSC) proliferation and stemness maintenance. (A) Detection of SUMO1 and SUMO2/3 levels in covalent and free states by western blot analysis. (B) Cell morphological changes in various experimental groups (scale bar, $25 \mu \mathrm{m}$ ). Arrows indicate aging cells with enlarged and flattened cell bodies and an increased volume of nuclei and nucleoli. (C) Growth curves of BMSCs in various groups. (D) Cell cycle of BMSCs in various experimental groups. (E) Detection of nerve cells differentiated from BMSCs in various groups by immunofluorescence (scale bar, $10 \mu \mathrm{m}$ ).

of protein SUMOylation caused by exogenous stress is the translocation of the SUMO complex from the cytoplasm to the nucleus $(14,17,20-22)$. We also observed a shift of SUMO1 and the SUMO2/3 complex from the cytoplasm to the nucleus with a decrease in incubation temperature (Fig. 2E).

SUMOs are essential for BMSC proliferation and stemness maintenance. To determine whether SUMOs are required for the proliferation and stemness maintenance of BMSCs, we used RNA interference to successfully knockdown the expression of SUMO1/2/3 in BMSCs (Fig. 3A). Upon the knockdown of SUMO1/2/3, the BMSCs almost stopped proliferating and most of the cells exhibited marked cell aging, evidenced by enlarged and flattened cell bodies, and an increased nucleus and nucleolar volume (Fig. 3B-D). Analysis of cell cycle changes by flow cytometry confirmed that a large number of cells was arrested at the G0/G1 phase, which was accompanied by a certain percentage of apoptotic cells (Fig. 3D). Cell differentiation experiments revealed that the BMSCs in which the SUMO1/2/3 gene was knocked down no longer maintained their stemness and differentiated toward neural cells (Fig. 3E).

Inhibition of Ubc9 blocks SUMO protein modification and reduces the tolerance of BMSCs to adverse environmental conditions. To clarify the role of SUMOylation in BMSC proliferation, differentiation and tolerance to adverse conditions, spectomycin $\mathrm{B} 1$, a reported $\mathrm{Ubc} 9$ inhibitor, was employed to prevent the binding of SUMOs to SUMO substrates. The results revealed that, unlike the effects of SUMO1/2/3 knockdown in
BMSCs, the inhibition of Ubc9 did not cause the rapid aging of the cells, but slowed the proliferation rate, and low temperatures appeared to further inhibit these processes (Fig. 4B-D). OGD induced the significant apoptosis of BMSCs. However, the inhibition of Ubc9 further induced apoptosis, resulting in a higher percentage of apoptotic cells (Fig. 4E and F), and higher levels of LDH (Fig. 4G).

PCNA, Oct4, p53 and HIF-1 $\alpha$ SUMOylation is regulated by low temperatures, and participates in BMSC proliferation, stemness maintenance and resistance to apoptosis. Based on previous studies (30-33), 4 proteins were investigated in this study. The relults of western blot revealed a decrease in the protein level of PCNA, while Oct4 expression increased with the decrease in temperature (Fig. 5A, C and D). However, when Ubc9 was suppressed, the protein levels of both PCNA and Oct4 were decreased significantly (Fig. 5A, C and D). In BMSCs, there was a small amount of p53 and almost no HIF-1 $\alpha$ expression under normal culture conditions (Fig. 5B, E and $F$ ). However, the protein expression levels of p53 and HIF-1 $\alpha$ were significantly increased in the OGD environment (Fig. 5B, E and F). This increasing trend was blocked by spectomycin B1 through the blocking of the binding of SUMOs to their target proteins (Fig. 5B, E and F).

\section{Discussion}

Therapeutic hypothermia is a potential treatment for traumatic brain injury $(8,10)$. Previous studies have suggest that, by 
A

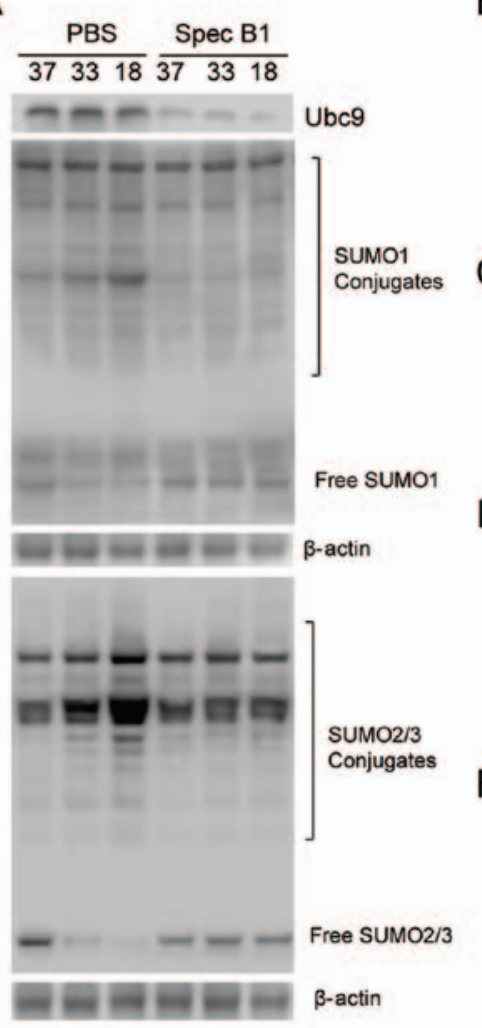

B

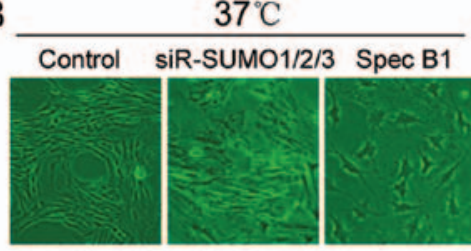

C

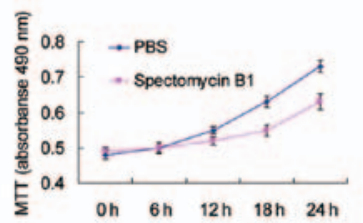

$\mathrm{E}$

PBS

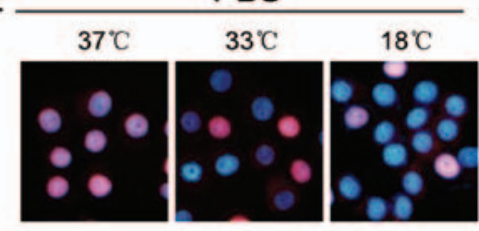

$\mathrm{F}$

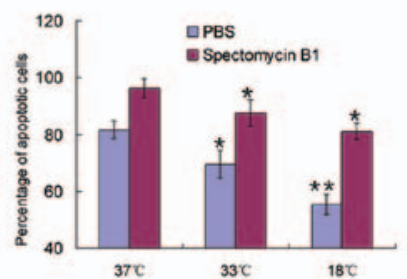

$18^{\circ} \mathrm{C}$

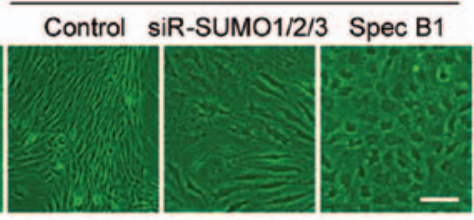

D $\overline{\mathrm{g}_{\mathrm{g}}} 0.7[\rightarrow 37 \mathrm{c}$

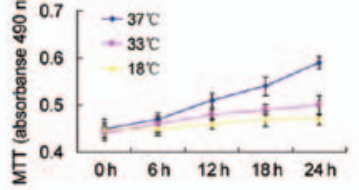

Spectomycin B1

\begin{tabular}{c|c|c}
\hline $37^{\circ} \mathrm{C}$ & $33^{\circ} \mathrm{C}$ & $18^{\circ} \mathrm{C}$ \\
\hline$\circ 0$ & $\circ$ & $\circ$ \\
$\circ .0$ & $\circ$ & $\circ$ \\
$\circ$ & $\circ$ & $\circ$ \\
\hline
\end{tabular}

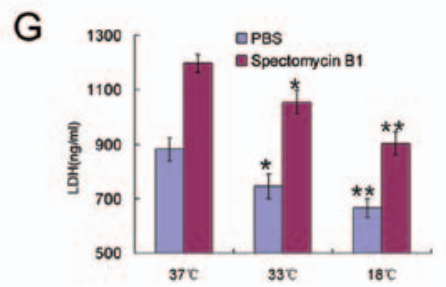

Figure 4. Inhibition of Ubc9 blocks small ubiquitin-like modifier (SUMO) protein modification and reduces bone marrow-derived mesenchymal stem cell (BMSC) tolerance to adverse environments. (A) Detection of the expression levels of Ubc9 as well as SUMO1 and SUMO2/3 in covalent and free states by western blot analysis. (B) Cell morphological changes in various experimental groups (scale bar, $25 \mu \mathrm{m}$ ). (C and D) Growth curves of BMSCs in various groups. (E) Cell cycle of BMSCs in various experimental groups. (E and F) Apoptosis detection by TUNEL assay (scale bar, $10 \mu \mathrm{m}$ ). Red, apoptotic cells; blue, all nuclei.

(G) Measurement of lactate dehydrogenase $(\mathrm{LDH})$ content by enzyme-linked immunosorbent assay (ELISA). ${ }^{*}<<0.05,{ }^{* *} \mathrm{p}<0.01$.

A

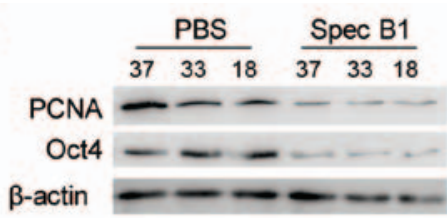

B

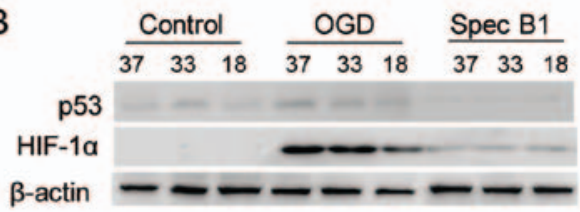

$\mathrm{F}$
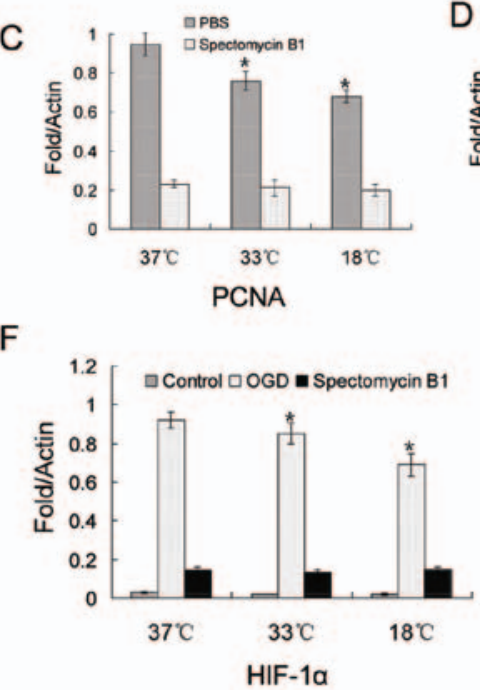
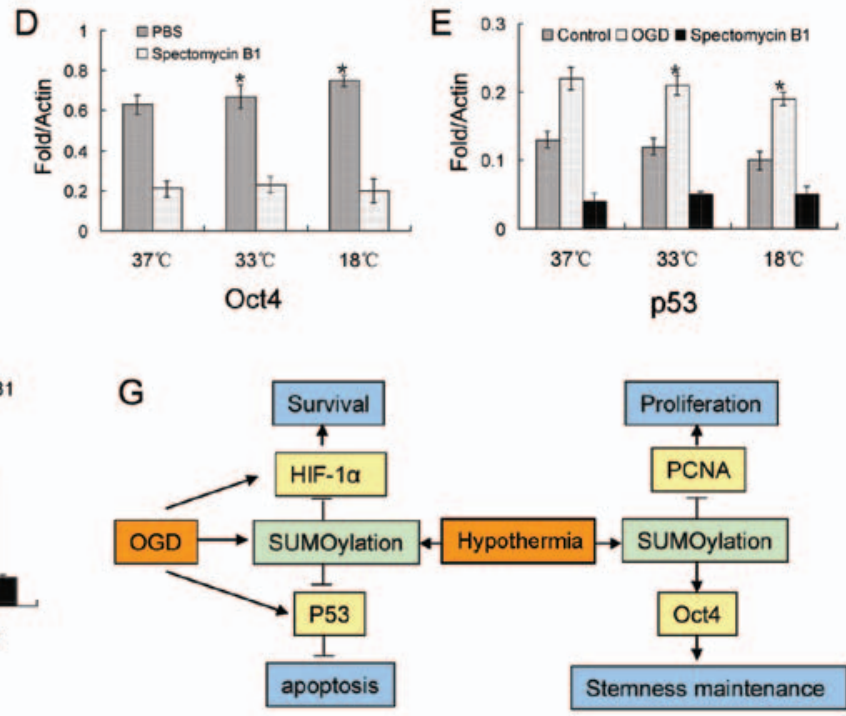

Figure 5. Proliferating cell nuclear antigen (PCNA), Oct4, p53 and hypoxia-inducible factor-1 $\alpha$ (HIF-1 $\alpha$ ) SUMOylation enhances bone marrow-derived mesenchymal stem cell (BMSC) proliferation, stemness maintenance and resistance to apoptosis. (A) Detection of PCNA and Oct4 expression levels by western blot analysis. (B) Detection of p53 and HIF-1 $\alpha$ expression levels by western blotting. (C and D) Quantification of data in A. "p $<0.05$, compared with PBS group. (E and F) Quantification of data in B. "p $<0.05$, compared with the control group. (G) A putative regulatory model of hypothermic induction of protein SUMOylation that enhances proliferation, stemness maintenance, and resistance to apoptosis in BMSCs. 
slowing destructive cellular processes, and reducing edema and intracranial pressure, hypothermia may improve neurological outcomes in patients with traumatic brain injury or ischemic stroke $(34,35)$. Furthermore, in patients with severe brain injury, treatment with mild hypothermia increases the survival rate of transplanted stem cells in the injured brain area $(11,12)$. However, the exact mechanisms involved remain unclear. In this study, we first investigated the effects of low temperature on the increase of BMSC tolerance to hypoxia in terms of SUMOylation, as it has been indicated that therapeutic hypothermia increases the SUMOylation of numerous proteins, which enhances the resistance of neurons to an adverse environment (36). By translation into clinical practice, such a strategy will further broaden the clinical treatment options and increase the confidence of doctors and patients in terms of treatment of brain injury.

First, we found that hypothermia increased the survival of BMSCs in a hypoxic environment. Furthermore, hypothermia was accompanied by very low cell proliferation and a decreased ability to differentiate into neural cells. To investigate whether the proliferative characteristics of BMSCs in a low temperature environment are dependent on SUMO protein modifications, an RNA interference plasmid, which silenced the expression of SUMO1/2/3 proteins, was transfected into the BMSCs. Our results revealed that BMSCs lacking SUMO1/2/3 expression did not proliferate and underwent rapid cell aging. This result indicates that SUMO1/2/3 are essential for the proliferation and survival of BMSCs. Furthermore, a reported Ubc9 inhibitor, spectomycin B1 (37), was employed to inhibit the binding of SUMO1/2/3 to SUMO substrates. Unlike the effects of SUMO1/2/3 knockdown in BMSCs, the inhibition of Ubc9 did not cause the rapid aging of the cells, but decreased the cell proliferation rate. This indicates that spectomycin B1 is more suitable for follow-up studies. Unlike known SUMOylation inhibitors, such as ginkgolic acid, spectomycin B1 binds directly to E2 (Ubc9) and selectively blocks the formation of the E2-SUMO intermediate (37). Our results revealed that spectomycin B1 effectively inhibited Ubc9. Moreover, the inhibition of Ubc9 effectively offset the increase in SUMOylation induced by treatment with hypothermia in the BMSCs. Upon he loss of the protective effect of SUMOylation on proteins, BMSC stemness maintenance was poor and they had a low tolerance against the same adverse environment. These results indicate that protein SUMOylation is essential for BMSC proliferation, stemness maintenance and enhanced tolerance to adverse conditions.

To delineate the underlying molecular mechanisms, we investigated 4 reported SUMO target proteins, PCNA, Oct4, p53 and HIF-1 $\alpha$ (30-33). PCNA, the DNA clamp for replicative polymerases, plays a central role in tje regulation of the cell cycle and their modification by ubiquitin (Ub) and SUMOs (38). In eukaryotic cells, PCNA is modified by Ub or Poly-Ub at lysine 164 (Lys164), and post-translational modifications of PCNA control the processing of replication intermediates (39). We found that hypothermia reduced the expression level of PCNA, and the inhibition of Ubc9 further decreased PCNA expression. This result is consistent with the very slow proliferation rate of BMSCs. The transcription factor, Oct4, is a master regulator that affects the fate of pluripotent stem cells and germ cell precursors (40). Oct4 expression is tightly regulated, and small changes in its expression level can have dramatic effects on differentiation and stemness maintenance (41). Oct4 is a target for SUMO-1 modification, and SUMOylation of Oct4 occurs at a single lysine, Lys118, located at the end of the aminoterminal transactivation domain and next to the Pit1-Oct-Unc86 (POU) DNA-binding domain (40). The SUMOylation of Oct4 significantly increases Oct4 stability and increases DNA binding (40). Our results revealed that, with the decrease in temperature, the expression level of Oct4 showed a gradually increasing trend. However, the inhibition of Ubc9 reduced the Oct4 expression level. These results suggest that Oct4 may be involved in the stemness maintenance of BMSCs. p53 is an antiproliferative transcription factor that increases the transcription rate of various genes involved in mitosis and apoptosis, which undergoes modification by SUMOylation (42). In this study, we found that a hypoxic environment induced the expression of p53 in BMSCs, but there was little effect on the BMSCs upon the inhibiton of Ubc9. Finally, we found that OGD conditions significantly induced the expression of HIF-1 $\alpha$, another protein that undergoes SUMOylation (43), and spectomycin B1 was able to offset this change. The above-mentioned results suggest a similar phenomenon in which SUMOs respond to various stresses by controlling multiple target proteins simultaneously to promote the proliferation and survival of BMSCs in adverse environments (Fig. 5G).

In conclusion, our results indicate the involvement of SUMOs and the SUMO pathway in the tolerance of BMSCs against adverse environments, and suggest a novel protective mechanism against hypothermia for BMSC survival. The results may lead to the future development of effective methods to improve the success rate of BMSC transplantation in adverse environments, such as brain injury and cerebral ischemia, to ultimately improve patient rehabilitation.

\section{Acknowledgements}

This study was supported by the National Natural Science Foundation of China (no. 81471175), and the Tianjin Health Bureau Science and Technology Projects (no. 2014KY23).

\section{References}

1. Russo MV and McGavern DB: Inflammatory neuroprotection following traumatic brain injury. Science 353: 783-785, 2016.

2. Masoudi MS, Rezaee E, Hakiminejad H, Tavakoli M and Sadeghpoor T: Cisternostomy for management of intracranial hypertension in severe traumatic brain injury; case report and literature review. Bull Emerg Trauma 4: 161-164, 2016.

3. Limpastan K, Norasetthada T, Watcharasaksilp W and Vaniyapong T: Factors influencing the outcome of decompressive craniectomy used in the treatment of severe traumatic brain injury. J Med Assoc Thai 96: 678-682, 2013.

4. Shi J, Han P and Kuniyoshi SM: Cognitive impairment in neurological diseases: lessons from apolipoprotein E. J Alzheimers Dis 38: 1-9, 2014.

5. Benarroch EE: Microglia: multiple roles in surveillance, circuit shaping, and response to injury. Neurology 81: 1079-1088, 2013.

6. Chang EH, Adorjan I, Mundim MV, Sun B, Dizon ML and Szele FG: Traumatic brain injury activation of the adult subventricular zone neurogenic niche. Front Neurosci 10: 332, 2016.

7. Pietrini D, Piastra M, Luca E, Mancino A, Conti G, Cavaliere F and De Luca D: Neuroprotection and hypothermia in infants and children. Curr Drug Targets 13: 925-935, 2012.

8. Ahmed AI, Bullock MR and Dietrich WD: Hypothermia in traumatic brain injury. Neurosurg Clin N Am 27: 489-497, 2016.

9. Lazaridis C and Robertson CS: Hypothermia for increased intracranial pressure: is it dead? Curr Neurol Neurosci Rep 16: 78, 2016. 
10. Leshnower BG, Myung RJ, Thourani VH, Halkos ME, Kilgo PD, Puskas JD and Chen EP: Hemiarch replacement at $28^{\circ} \mathrm{C}$ : an analysis of mild and moderate hypothermia in 500 patients. Ann Thorac Surg 93: 1910-1916, 2012.

11. McAdams RM and Juul SE: Neonatal encephalopathy: update on therapeutic hypothermia and other novel therapeutics. Clin Perinatol 43: 485-500, 2016.

12. Dailey T, Mosley Y, Pabon M, Acosta S, Tajiri N, van Loveren $\mathrm{H}$, Kaneko Y and Borlongan CV: Advancing critical care medicine with stem cell therapy and hypothermia for cerebral palsy. Neuroreport 24: 1067-1071, 2013.

13. Wimmer P, Schreiner S and Dobner T: Human pathogens and the host cell SUMOylation system. J Virol 86: 642-654, 2012.

14. Kumar A and Zhang KY: Advances in the development of SUMO specific protease (SENP) inhibitors. Comput Struct Biotechnol J 13: 204-211, 2015.

15. Wilson VG: Sumoylation at the host-pathogen interface. Biomolecules 2: 203-227, 2012.

16. Swatek KN and Komander D: Ubiquitin modifications. Cell Res 26: 399-422, 2016.

17. Ulrich HD: Ubiquitin and SUMO in DNA repair at a glance. $J$ Cell Sci 125: 249-254, 2012.

18. Craig TJ and Henley JM: Protein SUMOylation in spine structure and function. Curr Opin Neurobiol 22: 480-487, 2012.

19. ChangE and Abe J:Kinase-SUMO networks in diabetes-mediated cardiovascular disease. Metabolism 65: 623-633, 2016.

20. Yang W, Ma Q, Mackensen GB and Paschen W: Deep hypothermia markedly activates the small ubiquitin-like modifier conjugation pathway; implications for the fate of cells exposed to transient deep hypothermic cardiopulmonary bypass. J Cereb Blood Flow Metab 29: 886-890, 2009.

21. Yang W and Paschen W: SUMO proteomics to decipher the SUMO-modified proteome regulated by various diseases. Proteomics 15: 1181-1191, 2015.

22. Wang L, Ma Q, Yang W, Mackensen GB and Paschen W: Moderate hypothermia induces marked increase in levels and nuclear accumulation of SUMO2/3-conjugated proteins in neurons. J Neurochem 123: 349-359, 2012.

23. Yamasaki S, Mera H, Itokazu M, Hashimoto Y and Wakitani S Cartilage repair with autologous bone marrow mesenchymal stem cell transplantation: review of preclinical and clinical studies. Cartilage 5: 196-202, 2014.

24. Mezey É and Nemeth K: Mesenchymal stem cells and infectious diseases: smarter than drugs. Immunol Lett 168: 208-214, 2015.

25. Ross CL, Siriwardane M, Almeida-Porada G, Porada CD, Brink P, Christ GJ and Harrison BS: The effect of low-frequency electromagnetic field on human bone marrow stem/progenitor cell differentiation. Stem Cell Res (Amst) 15: 96-108, 2015.

26. Lynch K and Pei M: Age associated communication between cells and matrix: a potential impact on stem cell-based tissue regeneration strategies. Organogenesis 10: 289-298, 2014.

27. Błogowski W, Bodnarczuk T and Starzyńska T: Concise review: pancreatic cancer and bone marrow-derived stem cells. Stem Cells Transl Med 5: 938-945, 2016.

28. Yang Z, Zhu L, Li F, Wang J, Wan H and Pan Y: Bone marrow stromal cells as a therapeutic treatment for ischemic stroke. Neurosci Bull 30: 524-534, 2014.
29. Liu Z, Jiang Z, Huang J, Huang S, Li Y, Yu S, Yu S and Liu X: miR-7 inhibits glioblastoma growth by simultaneously interfering with the PI3K/ATK and Raf/MEK/ERK pathways. Int J Oncol 44: 1571-1580, 2014.

30. Parker JL and Ulrich HD: A SUMO-interacting motif activates budding yeast ubiquitin ligase Rad18 towards SUMO-modified PCNA. Nucleic Acids Res 40: 11380-11388, 2012.

31. Tahmasebi S, Ghorbani M, Savage P, Gocevski G and Yang XJ: The SUMO conjugating enzyme Ubc9 is required for inducing and maintaining stem cell pluripotency. Stem Cells 32: 1012-1020, 2014

32. Stehmeier P and Muller S: Regulation of p53 family members by the ubiquitin-like SUMO system. DNA Repair (Amst) 8: 491-498, 2009.

33. Lee YJ, Bernstock JD, Nagaraja N, Ko B and Hallenbeck JM: Global SUMOylation facilitates the multimodal neuroprotection afforded by quercetin against the deleterious effects of oxygen/glucose deprivation and the restoration of oxygen/glucose. J Neurochem 138: 101-116, 2016.

34. Sadaka F and Veremakis C: Therapeutic hypothermia for the management of intracranial hypertension in severe traumatic brain injury: a systematic review. Brain Inj 26: 899-908, 2012.

35. Shankaran S: Current status of hypothermia for hypoxemic ischemia of the newborn. Indian J Pediatr 81: 578-584, 2014.

36. Lee YJ and Hallenbeck JM: SUMO and ischemic tolerance. Neuromolecular Med 15: 771-781, 2013.

37. Hirohama M, Kumar A, Fukuda I, Matsuoka S, Igarashi Y, Saitoh H, Takagi M, Shin-ya K, Honda K, Kondoh Y, et al: Spectomycin B1 as a novel SUMOylation inhibitor that directly binds to SUMO E2. ACS Chem Biol 8: 2635-2642, 2013.

38. Dieckman LM, Freudenthal BD and Washington MT: PCNA structure and function: insights from structures of PCNA complexes and post-translationally modified PCNA. Subcell Biochem 62: 281-299, 2012

39. Parker JL and Ulrich HD: In vitro PCNA modification assays. Methods Mol Biol 920: 569-589, 2012.

40. Wei F, Schöler HR and Atchison ML: Sumoylation of Oct4 enhances its stability, DNA binding, and transactivation. J Biol Chem 282: 21551-21560, 2007.

41. Yang F, Yao Y, Jiang Y, Lu L, Ma Y and Dai W: Sumoylation is important for stability, subcellular localization, and transcriptional activity of SALL4, an essential stem cell transcription factor. J Biol Chem 287: 38600-38608, 2012.

42. Laura MV, de la Cruz-Herrera CF, Ferreirós A, Baz-Martínez M, Lang V, Vidal A, Muñoz-Fontela C, Rodríguez MS, Collado M and Rivas C: KSHV latent protein LANA2 inhibits sumo2 modification of p53. Cell Cycle 14: 277-282, 2015.

43. Wang F, Cai F, Shi R, Wei JN and Wu XT: Hypoxia regulates sumoylation pathways in intervertebral disc cells: implications for hypoxic adaptations. Osteoarthritis Cartilage 24: 1113-1124, 2016.

This work is licensed under a Creative Commons Attribution-NonCommercial-NoDerivatives 4.0 International (CC BY-NC-ND 4.0) License. 